\title{
Reflexões sobre o uso das tecnologias móveis como uma ferramenta auxiliar ao ensino
}

Reflections on the use of mobile technologies as an auxiliary tool to teaching

\author{
A. G. Cronemberger ${ }^{1 *}$; N. N. Soares²; L. M. Gomes ${ }^{2}$; F. C. L. Ferreira ${ }^{2}$; J. G. S. L. \\ Junior $^{1}$ \\ ${ }^{\text {I}}$ Faculdade de Física/Instituto de Ciências Exatas/Programa Nacional de Mestrado Profisional em Ensino de Física, \\ Universidade Federal do Sul e Sudeste do Pará, 68507-590, Marabá - Pará, Brasil \\ ${ }^{2}$ Faculdade de Matemática/Instituto de Ciências Exatas, Universidade Federal do Sul e Sudeste do Pará, CEP 68507 - \\ 590, Marabá - Pará, Brasil
}

*kbergnet@yahoo.com.br

(Recebido em 19 de dezembro de 2016; aceito em 05 de janeiro de 2017)

Este artigo é uma pesquisa sobre o uso dos dispositivos móveis no ambiente escolar. São apresentados dados que corroboram a utilização destes dispositivos em sala de aula como ferramenta de aprendizagem, dada a sua utilização massiva na sociedade, com números bastante expressivos na faixa etária dos jovens e adolescentes. São feitas análises sobre sua utilização versus o uso de desktops e laboratórios de ensino. Também são feitas discussões sobre a aprendizagem móvel (mobile learning). Nesse contexto são destacados os desafios enfrentados pelos professores quanto à sua utilização em sala de aula, bem como as perspectivas e tensões que cercam o uso de aparelhos móveis como objeto de aprendizagem. Além disso, buscou-se entender o porquê do uso da tecnologia no ambiente escolar ser bastante promissor. Finalmente, são comentadas a utilização de dispositivos móveis com simulações virtuais e a gameficação no ensino. Palavras-chave: Dispositivos Móveis; Tecnologias de Informação e Comunicação; Aprendizagem Móvel.

This paper is a survey about the use of mobile devices in the school environment. Data are presented that corroborate the use of these devices in the classroom as a learning tool, because of their massive use in society, with very significant numbers in the youth age group. Analyzes are done on its use versus the use of desktops and teaching labs. There are also discourses about mobile learning. In this context are highlighted the challenges faced by teachers regarding their use in the classroom, as well as the perspectives and tensions surrounding the use of mobile devices as an object of learning. In addition, we sought to understand why the use of technology in the school environment is very promising. Finally, the use of mobile devices for the realization of virtual simulations and gamefication in teaching are discussed. Keywords: Mobile devices; Information and Communication Technologies; Mobile Learning.

\section{INTRODUÇÃO}

O trabalho do professor no ambiente de sala de aula é algo desafiador. Frequentemente, os docentes trazem a tona queixas do tipo: "os alunos não têm interesse", "aluno de hoje em dia não quer mais estudar, só vive com o fone de ouvido", "Aluno só quer saber de celular", assim por diante.

Buscando-se soluções para resolver problemas desta natureza, a escola e o Estado, cada vez mais têm demonstrado esforços com intuito de amenizar ou inibir o uso dos aparelhos celulares nas dependências da escola - suposto responsável pelos déficits de aprendizagem.

No Brasil, na maioria dos Estados não é mais permitida, em sala de aula, a utilização de celulares ou aparelhos eletrônicos. Aliás, há um projeto de Lei Federal (2246-A, de 2007, apenso PL número 3486 de 2008) que trata deste tema. Segundo GIL (2013) [1], esse tipo de proibição visa coibir fraudes nos processos avaliativos e a falta de atenção nas aulas que leva a queda do rendimento escolar.

Entretanto, pesquisas apontam que essa nova geração apresenta potencialidades e peculiaridades diferentes das gerações anteriores, quanto à interatividade com as novas tecnologias. SEABRA (2010) [2] afirma que estudos e pesquisas que foram realizados nos 
últimos 20 anos sobre a interação das crianças e dos jovens com as tecnologias digitais permitem comprovar que uma nova inteligência está se desenvolvendo nas novas gerações que crescem incluídas na cultura digital.

Em pleno século XXI, fica difícil para os jovens se desconectarem da tecnologia em prol da educação tradicional. Segundo JAYAKUMAR (2008) [3], estudos indicam que muitos jovens já possuem "nomofobia", que é a fobia causada pelo desconforto ou angústia resultante da incapacidade de comunicação através de aparelhos celulares ou computadores. O temo nomofobia vem da expressão inglesa "no-mobile-phone phobia", ou seja, é o medo de estar desprovido de um celular. De acordo com estudos recentes, $66 \%$ da população britânica sofrem deste distúrbio e, $44 \%$ tem pelo menos dois telemóveis [4].

Assim, fica evidente que o ensino precisa caminhar lado a lado com a informatização e a tecnologia, de modo que possa permitir o estudo em classe e extraclasse com o auxilio de tais aparatos tecnológicos e possa preparar o estudante para a realidade que irá encontrar, quando de fato, conhecimentos e habilidades são necessários para lidar com situações análogas à realidade.

Fazer uso de um ambiente de ensino e aprendizagem com o empoderamento das novas tecnologias, em prol de estratégias de ensino aprendizagem que possibilitem extrair as potencialidades do aluno, ainda é desafiador para a grande maioria dos docentes. Assim, para TOTTI et al. (2001) [5], a tecnologia que antes era vista como algo que tirava o sujeito do convívio social, torna-se cada vez mais customizada de forma que os ambientes virtuais ganham um ponto muito positivo para a pedagogia centrada no aluno.

É justamente no âmbito da tecnologia que, de acordo com LEVY (1999) [6], é necessária uma mudança de métodos para a promoção de uma educação mais eficaz. Desse modo, já afirmava Albert Einstein que "Insanidade é continuar a fazer sempre a mesma coisa e esperar resultados diferentes".

Sendo assim, tem-se um paradigma sobre os dispositivos eletrônicos móveis (smartphones, tablets, ipods). Um entrave na presença do ensino ou um trunfo de escape para uma aprendizagem significativa?

Segundo VALENTE (1997) [7], o computador pode ser usado na educação como máquina de ensinar ou como máquina para ser ensinada. $\mathrm{O}$ uso do computador como máquina de ensinar consiste na informatização dos métodos de ensino tradicionais. Do ponto de vista pedagógico esse é o paradigma instrucionista, acrescenta o autor.

Nesse contexto, não é recente a ideia de usar dispositivos tecnológicos na educação. No final dos anos cinquenta do século passado, Skinner propôs o ensino por meio do uso da tecnologia disponível na época. Eram as famosas "máquinas de ensinar", que nada mais eram do que programas com vários exercícios que deveriam ser respondidos individualmente pelos alunos. Nesta prática de Skinner, as questões eram respondidas logo após pela máquina, promovendo assim a chamada "função da mudança de comportamento" destaca CARVALHO (2009) [8].

Além disso, essa mudança comportamental contempla também a prática docente. De acordo com GADOTTI (2003) [9], o professor deixará de ser um lecionador para ser um organizador do conhecimento e da aprendizagem, um mediador do conhecimento, um aprendiz permanente, um construtor de sentidos, um cooperador e, sobretudo, um organizador da aprendizagem.

\section{MATERIAL E MÉTODOS}

O presente trabalho foi desenvolvido a partir de pesquisas realizadas sobre o "estado da arte" do tema em questão. De acordo com FERREIRA (2002) [10], esse tipo de trabalho tem por objetivo realizar levantamento do que se conhece sobre um determinado assunto a partir de estudos e análises feitas em uma determinada área.

Assim, buscou-se fazer um levantamento de trabalhos sobre a presente linha de pesquisa e fazer uma análise sobre o tema, buscando sua melhor compreensão. Segundo o referido pesquisador [10], as pesquisas de caráter bibliográfico, com o objetivo de inventariar e sistematizar a produção em determinada área do conhecimento, (chamadas, usualmente, de pesquisas do "estado da arte"), (...) são, sem dúvida, de grande importância, pois podem conduzir à plena compreensão do estado atingido pelo conhecimento a respeito de determinado tema - sua amplitude, tendências teóricas, vertentes metodológicas.

Desta maneira, objetivou-se enfocar trabalhos que pudesse demonstrar tendências e possibilidade de pesquisa dentro da temática do presente artigo. 


\section{RESULTADOS E DISCUSSÃO}

\subsection{Dispositivos Móveis x Desktops e Laboratórios}

A sociedade está em constante transformação e autocriação, onde todos os campos, sejam eles cultural, educacional ou político, fluem em constantes mudanças buscando suas readequações [11]. Dentre todas essas transformações, cabe destacar a rapidez com que as Tecnologias de Informação e Comunicação (TIC's) se espalharam. Pesquisas organizadas pelo Comitê Gestor da Internet no Brasil [12] atestam este fato e destacam o significativo aumento do uso de dispositivos móveis na faixa etária dos jovens.

Para PONTE (2000) [13], dentro de um contexto internacional, as TIC's correspondem a todas as tecnologias que proporcionam através de softwares ou hardwares, a autocomunicação nos processos de negócios, pesquisas e ensino-aprendizagem.

Segundo o relatório (Fig. 1) da TELECO (2013)[14], empresa de consultoria em inteligência e telecomunicações, $93 \%$ dos alunos de ensino médio possuem celular, e quase metade destes tem acesso à internet através do dispositivo móvel. Outro dado surpreendente é que no Brasil, em novembro de 2014 , havia a surpreendente quantia de 280,5 milhões de celulares, ou seja, $40 \%$ mais celulares do que o número de habitantes.

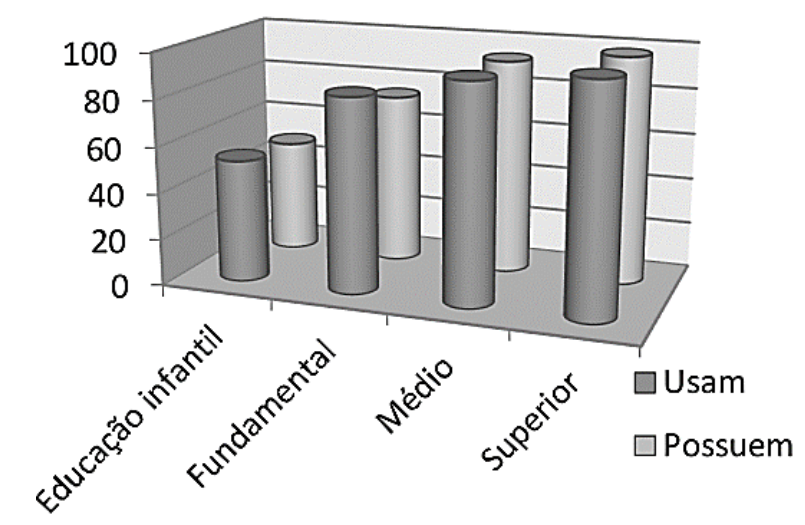

Figura 1: Percentual de alunos que usam e possuem celulares.

Fonte: TELECO 2013

Por outro lado, de acordo com a pesquisa realizada pela CGIBR (2012) [12], 59\% dos entrevistados declararam ter em algum momento de suas vidas utilizado um desktop. Estes chegaram aos consumidores muito antes dos celulares, mas não foram capazes de se difundirem tanto quando os celulares.

O número de pessoas que utilizaram em algum momento de suas vidas um computador não é tão superior ao número daqueles que possuem computador em suas residências.

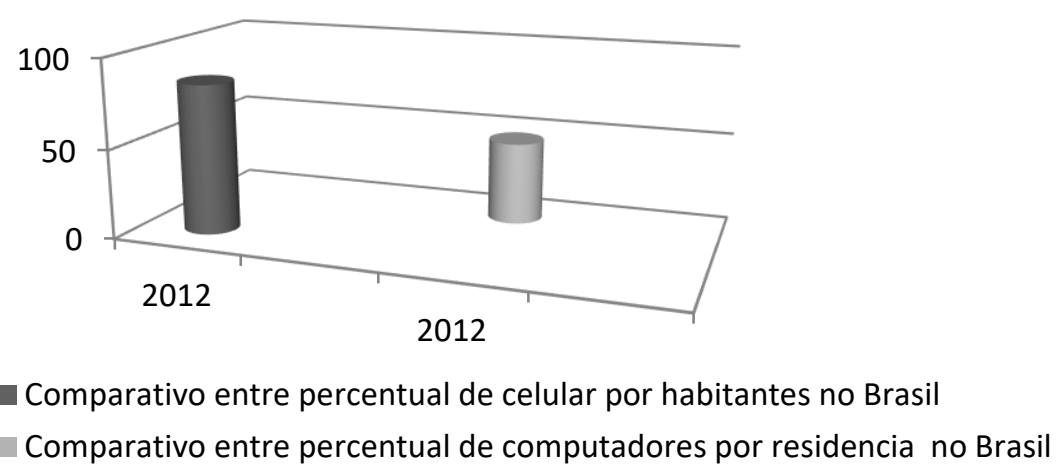

Figura 2: Percentual da população que possui celular em relação a percentual da população que possui computador no Brasil. 
A Figura 2 foi obtida a partir dos dados de CAVALCANTE (2014) [15] e de TELECO (2014) [14] e mostra o comparativo entre o percentual por habitante e por residência para celulares e computadores, respectivamente.

A Figura 3 mostra o gráfico do percentual de escolas com laboratórios de informática e de pessoas que possuem celular, sendo ambos os percentuais dados por região. $\mathrm{O}$ gráfico foi obtido a partir de dados do INEP (2013) [16] e da TELECO (2013) [14]. Quando se compara esses percentuais, verifica-se de imediato que o percentual de pessoas com celular por região é maior que o percentual de escolas com laboratórios de informática. Um outro aspecto interessante a ser observado, é que no caso dos dispositivos móveis, a distribuição percentual por região é praticamente homogênea, ou seja, superam em relação a distribuição percentual dos laboratórios de informática nas escolas (computadores disponibilizados para a mediação de práticas de ensino) de forma mais igualitária, já que os percentuais para todas as regiões são muito próximos. É evidente que as regiões norte e nordeste estão desestruturadas de laboratórios de informática, o que resulta na ausência de práticas de ensino de TIC's no que tange aos computadores escolares.

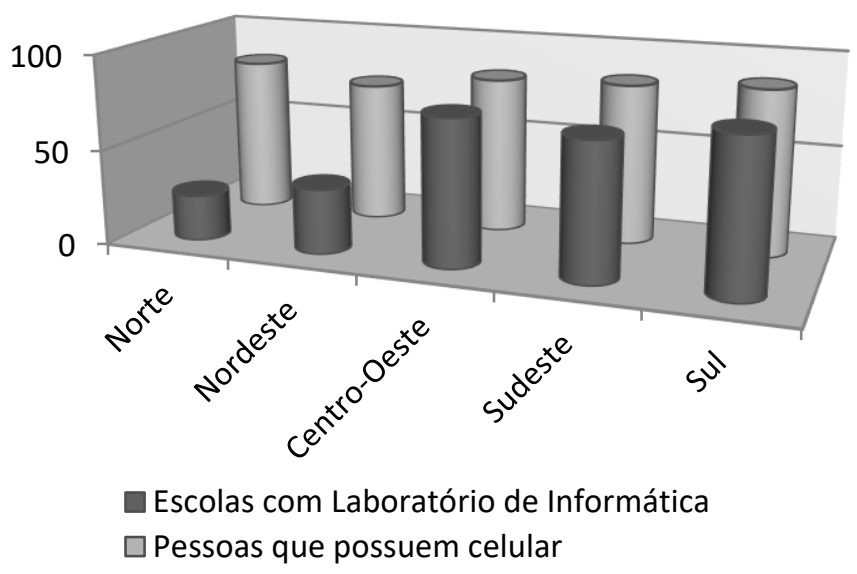

Figura 3: Percentual da população que possui celular em relação a percentual das escolas com laboratório de informática no Brasil

Ainda, segundo dados do INEP (2013) [16], apenas 48,6\% das escolas no país possuem laboratório de informática e, conforme pode ser deduzido a partir da (Fig. 3), essa distribuição nas regiões do país se dá de forma muito desigual, diferentemente do percentual dos usuários de celulares. Assim, fica evidente que na maioria dos espaços de ensino, é possível contar com um maior acesso a dispositivos moveis de alunos do que com os laboratórios de informáticas em operacionalidade nas escolas, principalmente no que tange a uma distribuição regional igualitária.

Um ponto positivo quanto a tecnologia empregada nos celulares, é que estes oferecem uma gama de funções, despertando assim, o interesse nos discentes. Estes procuram adquirir os modelos mais atuais. Entres os mais variados modelos e funções disponíveis, destaca-se o Smartphone, aparelho capaz de executar as mais variadas tarefas. Através de seus diversos sensores instalados eles são capazes de executar as mais diversas funções. Outra característica importantíssima que justifica a utilização de Smartphone em atividades de ensino em sala de aula é sua portabilidade, fáceis de manuseio e deslocamento sem restrições de espaço.

Atualmente, no mercado estes dispositivos além é claro da minimização do custo ao longo do tempo, são equipamentos com câmera, bluetooth, sensores de temperatura, velocidade, acelerômetro, microfone, giroscópio, luxímetros, GPS e outros sensores.

Com mais de 5,9 bilhões de assinaturas de telefones celular no mundo (UNESCO, 2014) [17] fica evidente que estes dispositivos podem ser vislumbrados como uma potente ferramenta de ensino. Assim, educadores necessitam estar habilitados para a utilização destas novas tecnologias, que certamente vão dar suporte a eles na implementação das atividades pedagógicas escolares, possibilitando desdobramentos no desenvolvimento de novas competências e habilidades, inserindo assim, dentro do contexto escolar, novas situações de ensino e aprendizagem.

\subsection{M-Learning}

Com os avanços da informática aumentam as ferramentas para aplicações no ensinoaprendizagem. Daí, surgem os Ambientes Virtuais de Aprendizagem (AVAs). Tais ambientes se 
apresentam na academia como uma opção tecnológica para atender as demandas e necessidades exigidas do ensino, já que o AVA consiste em um conjunto de recursos tecnológicos de comunicação e informação em ciberespaços, que veicula conteúdos e permite interação entre os envolvidos no processo educacional.

Para MILLIGAN (1999) [18], o termo AVA deve ser usado para descrever um software baseado em um servidor e modelado para gerenciar e administrar os variados aspectos da aprendizagem, como disponibilizar conteúdos, acompanhar o estudante, avaliar o processo de ensino-aprendizagem, dentre outros. No entanto, não tem se verificado um número crescente de pesquisas na proposta do AVA dentro do cenário de ensino aprendizagem em dispositivos móveis.

Com o avanço das tecnologias digitais, as TIC's possibilitam um grande campo de pesquisas em ensino em dispositivos móveis. Dentre esses campos, surge o conceito do m-learning.

Segundo DOCHEV (2006) [19] a m-learning ou Mobile Learning (Aprendizagem Móvel) é considerado um paradigma emergente, por seu diferencial em relação a outras tecnologias eletrônicas (e-learning) devido ao seu aprendizado móvel.

Apesar de muitos acreditarem no potencial crescimento em pesquisas e práticas no $\mathrm{M}$ Learning, estudos feitos por ALMEIDA (2013) [20], revelam que as pesquisas aqui no Brasil não se dão de forma linear, mas em picos em alguns momentos, sendo que no último ano da pesquisa feita pelo referido autor, o m-learning terminou em queda considerável. A Figura 4 mostra os resultados desta pesquisa no período 2003-2012.

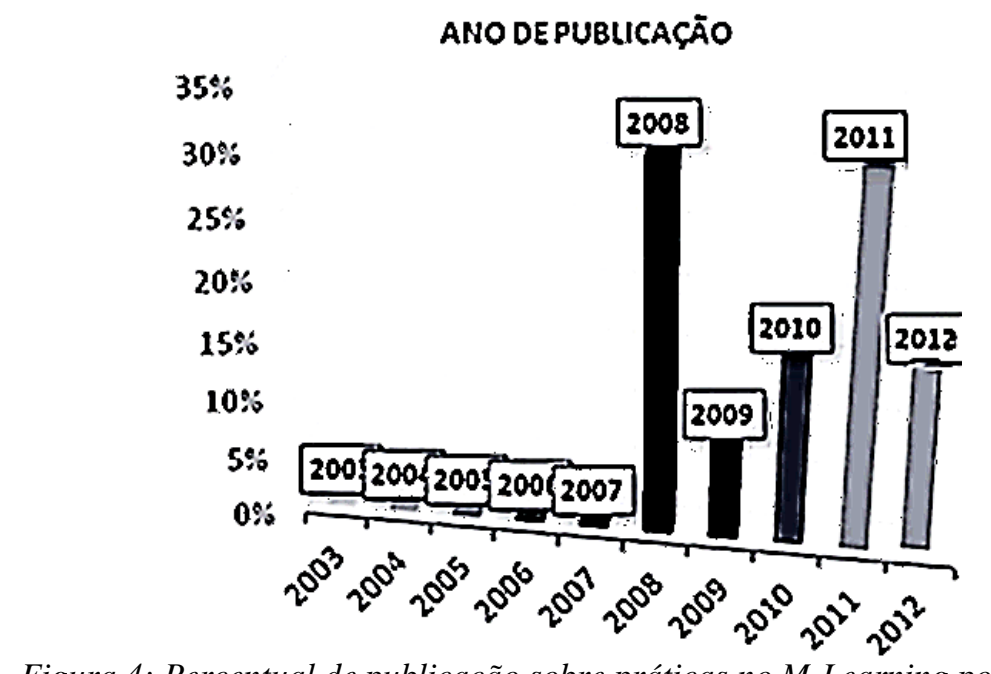

Figura 4: Percentual de publicação sobre práticas no M-Learning por ano.

Fonte: ALMEIDA (2013) [20] com modificações.

Apesar dos altos e baixos das pesquisas no M-Learning, por outro lado, é importante destacar que a UNESCO (2014) [17] calculou os gastos globais anuais (2014) em produtos e serviços de aprendizagem móvel para um valor de 3,4 bilhões de dólares americanos por ano. Mesmo no Brasil, segundo SALDANA (2012) [21], pelo menos 150 escolas particulares no país iniciaram seu ano letivo em 2013 com um sistema de ensino por meio de utilização de tablets.

Fica evidente que, apesar das oscilações na pesquisa e prática do M-Learning, o mercado dos dispositivos móveis está se popularizando cada vez mais. Como exemplo, destaca-se o caso do sistema operacional Android, utilizado em dispositivos móveis, onde em 2014, a Google atingiu a marca de um bilhão de usuários ativos por mês no mundo [22].

\subsection{Dificuldades de implementação para M-Learning}

Alguns pontos podem ser destacados para a falta de disseminações das pesquisas em mlearning, resultando na baixa produção e queda nos últimos anos: O primeiro ponto está associado ao fato de que muitos docentes ainda conservam suas mistificações e falsas crenças de que o uso do celular pelos alunos é um entrave, que proporciona dispersão e desmotivação nas aulas e no ensino. Tal pensamento é totalmente oposto aquilo que os pesquisadores YAY et al. (2010) [23] afirmam, onde, segundo eles, o mobile learning possibilita um ensino que favorece a estudantes e professores uma aprendizagem mais significativa. 
O segundo ponto está relacionado ao fato de que o uso de celulares em sala de aula ser proibido por lei. Quem vai se interessar em estimular o uso de um dispositivo que é proibido. Certamente, isto inibe alguns potenciais pesquisadores. Outra possibilidade está relacionada ao fato de muitos professores serem leigos a respeito da utilização de M-Learning no ensino.

Outro ponto refere-se aos resultados negativos bastantes significativos obtidos na verificação de aprendizagem por meio da utilização do M-Learning. A (Fig. 5) apresenta um gráfico que demostra que a aprendizagem com o uso do M-Learning é mínima, ou seja, algo em torno de $20 \%$.

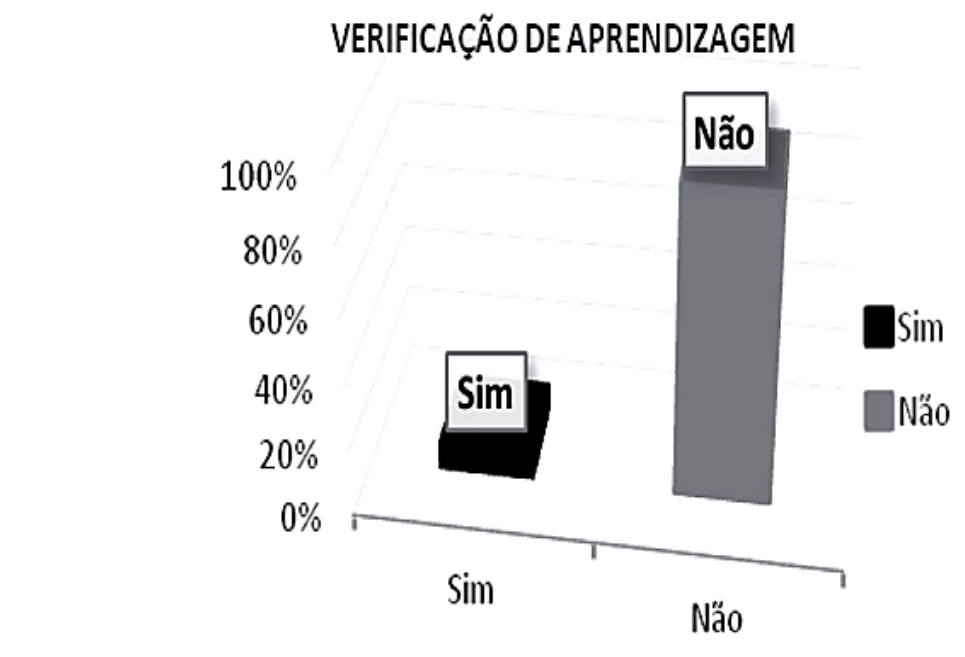

Figura 5: Percentual de aprendizagem por meio da utilização do M-Learning.

Fonte: ALMEIDA (2014) com modificações.

Com base nos pontos apresentados, pode se constatar que isso de fato remete a uma falsa realidade sobre a potencialidade do m-learning, mas acredita-se que o motivo está relacionado as técnicas (ou mesmo a falta delas) que estão sendo empregadas de modo equivocado. Segundo CLARK (2002) [24], "...a história está cheia de tentativas fracassadas de revolucionar aprendizagem por meio de inovações tecnológicas. Felizmente, estas tentativas nos ensinaram uma lição importante: para a tecnologia poder melhorar a aprendizagem, ela precisa 'se encaixar na vida do estudante... e não vice-versa. ...".

Quando se busca estabelecer uma atividade dentro de sala de aula é fundamental ter uma atividade afetivo-motivacional [25], e este resultado depende de quão atrativo é para a mentalidade do aluno e não para a concepção do professor. Não basta fazer as mesmas coisas com o único diferencial de que está utilizando algum dispositivo eletrônico.

É fato que os celulares são uma preferência quase absoluta das pessoas. É interessante que sejam feitos levantamentos no sentido de identificar aplicativos que as pessoas mais utilizam nos celulares e o porquê de sua utilização. GUGELMIN (2013) [26] destaca que os jogos eletrônicos (tanto gratuitos como os pagos) são recordistas pela preferencia dos usuários correspondendo a $40 \%$ dentre todas as categorias. Segundo este autor, isso corresponde a $80 \%$ da lucratividade do Google Play ${ }^{\circledR}$.

Nessa perspectiva, entende-se que os jovens querem um ensino dinâmico, lúdico e que seja sobre uma realidade envolvente. Segundo SILVEIRA et al. (1998) [27], os jogos podem ser empregados em uma variedade de propósitos dentro do contexto de aprendizado. Um dos usos básicos e muito importantes é a possibilidade de construir-se a autoconfiança. Outro é o incremento da motivação, além do mais, conclui o autor que o uso de jogos no ensino torna-se um método eficaz que possibilita uma prática significativa daquilo que está sendo aprendido. Até mesmo o mais simplório dos jogos pode ser empregado para proporcionar informações factuais e praticar habilidades, conferindo destreza e competência - acrescenta.

O desafio, sem dúvida, é como utilizá-los no ambiente de sala de aula. Nesse sentido, é oportuno destacar a gamificação como uma estratégia a ser utilizada no ambiente escolar. A gamificação traz para os alunos em sala de aula o lado lúdico dos games e o esquema de bonificações, o que pode ser muito motivador.

Apesar da grande expectativa de pesquisas no Brasil no que se espera para o futuro sobre a área da M-Learning, observa-se que os maiores desenvolvedores de soluções ou práticas em mlearning do país estão em centro acadêmicos. Apesar de que há pouco investimento por parte de 
organizações empresariais em iniciativas de aplicação de m-learning, segundo destaca ZANELLA et al. (2014) [28], é um mercado que está se desenvolvendo e cada vez mais crescendo no país.

\section{CONCLUSÃO}

$\mathrm{Na}$ atualidade, é nítida e preocupante a situação em que se encontra a educação brasileira. Fatores políticos, econômicos e sociais, dentre outros, contribuem para este caos. Destacam-se também a dificuldade que os docentes enfrentam em suas práticas didático-pedagógicos e alunos desprovidos de atenção e estímulos às aulas, em função dos mais variados problemas. Tais dificuldades são patentes, principalmente na assimilação do conteúdo e na conexão de tais assuntos, tornando a prática docente uma tarefa muito árdua.

Torna-se inevitável não pensar, em meio aos diversos problemas que vem, cada vez mais, roubando a atenção dos alunos dentro da sala de aula, no uso inadequado dos aparelhos celulares. Entretanto, não se pode negar que as tecnologias, sejam elas por meio de computadores, TV ou celulares, já fazem parte do cotidiano dos alunos.

Nesse aspecto, considera-se que os celulares são populares, fáceis no manuseio, com preço acessível, seus aplicativos vêm aos poucos se tornando potentes complementos para o desenvolvimento do trabalho docente. Enfim, porque não torna-los uma ferramenta auxiliar ao ensino como forma de estudo? Ao contrário de extingui-lo da escola?

A escola, não pode ignorar o desenvolvimento tecnológico e uso desses dispositivos no processo ensino. Cabe à ela instruir a maneira adequada da utilização dessa ferramenta de ensinoaprendizagem, principalmente em disciplinas dotadas de conteúdos complexos e abstracionais, assim como são os conceitos da Física.

Nesse artigo, dentre os dados levantados, alguns mostraram fatores divergentes a implementação do ensino via m-learning. Estes concluíram que a maioria dos tralhados na verificação de aprendizagem em m-learning tiveram uma baixa porcentagem no que tange a resultados positivos.

Porém esses dados são refutados no que tange a potencialidade do m-learning, uma vez que se constatou que a maioria das pesquisas apontam para uma crescente procura a dispositivos móveis, e que na maioria dos periódicos os participantes alegam acreditar que os dispositivos móveis são uma boa ferramenta a ser utilizada no ensino.

Essa contestação se baseia em um novo ramo de pesquisa da pedagogia intitulada "pedagogia emocional", em que traz como resposta o fato de que a matriz cognitiva de todo ser humano está em suas emoções (CHABOT, 2013) [29]. Obviamente, não se pode negligenciar as análises das zonas associadas às emoções e à afetividade, pois são elas as chaves para o processo de aprendizado, sendo intransigível comungar que uma ferramenta como os dispositivos móveis que possuem tão grande apreço possa ser ineficiente como instrumento de aprendizagem.

Partindo-se do pressuposto que as limitações de fracasso estejam relacionadas às competências de ensino no processo cognitivo e não na inabilidade da utilização da ferramenta do Mobile Learning, acredita-se que as ferramentas de ensino são tão somente motivadoras para a promoção de estímulos emocionais e afetivos como facilitadores para a compreensão de novos saberes.

\section{REFERÊNCIAS BIBLIOGRÁFICAS}

1. Gil J. Lei proíbe uso de celular na sala de aula Disponível em: <http://gestaoescolar.abril.com.br/politicas-publicas/lei-proibe-uso-celular-sala-aula-739266.shtml> Acesso em: 17 Janeiro 2015.

2. Seabra C. Tecnologias na escola. Como explorar o potencial das tecnologias de informação e comunicação na aprendizagem. Porto Alegre: Telos Empreendimentos Culturais; 2010. 28p

3. Jayakumar A. Break free from Nomophobia, drunkorexia. Mid-day.com. http://www.midday.com/web/guest/lifeatwork/lifeatwork/ article?_EXT_5_arti1cleId=1071008\&_EXT_5_groupId=14. Acesso em 23 setembro de 2016.

4. Rosa P. Nomofobia, medo irracional de sair de casa sem telemóvel. Acesso em: <http://ricasaude.com/nomofobia-medo-irracional-de-sair-de-casa-sem-telemovel/>Acesso em: 17 Janeiro 2015. 
5. Totti AR, et al. M-Learning: Possibilidades para a Educação a Distância. Disponível em: <http://www.abed.org.br/congresso2011/cd/181.pdf>. Acesso em 18 Janeiro. 2015.

6. Levy P. Cibercultura. São Paulo, 1999. Disponível em: http://www.moodle.ufba.br/file.php/8897/levy_cibercultura.pdf. Acessoem 17 Janeiro 2015.

7. Valente JA. Informática na educação: instrucionismo x construcionismo. Educação Pública, 1997. Disponível em: <http://www.educacaopublica.rj.gov.br/biblioteca/tecnologia/0003.html> Acesso em: 18 Janeiro 2015.

8. Carvalho AB. Concepções de Aprendizagem e o Uso da Tecnologia na Educação a Distância: Das Máquinas de Ensinar ao Conceito de Aprendizagem Colaborativa. Encontro de Pesquisa Educacional do Norte e Nordeste, 2009, João Pessoa. Educação, Direitos Humanos e Inclusão Social. João Pessoa: Editora UFPB, 2009.

9. Gadotti M. Boniteza de um sonho: ensinar e aprender com sentido. Novo Hamburgo: Feevale.

10. Ferreira NSA. As pesquisas denominadas "estado da arte". Educação \& Sociedade, ano 23, n. 79, p. 257-272, ago. 2002.

11. Hargreaves A. O ensino na sociedade do conhecimento: educação na era da insegurança. Porto Alegre: Artmed, 2004.

12. CGIBR. Pesquisa sobre o uso das tecnologias da informação e comunicação no Brasil: TIC Domić́lios e TIC Empresas 2012. São Paulo: Comitê Gestor da Internet no Brasil, 2012. Disponível em: <www.cgi.br>. Acesso em 17 Janeiro 2015.

13. Ponte JP. Tecnologias de Informação e comunicação de professores: Que desafios? Revista Iberoamericana de Educación, Espanha, 24, p.63-90, 2000.

14. TELECO. Estatísticas de Celulares no Brasil. Perfil dos Usuários de Celular 2013. Disponível em http://www.teleco.com.br/ncel_usu.asp. Acessado em 18 Janeiro 2015

15. Cavalcante T. Quase metade dos domić́lios brasileiros tem computador, mostra Pnad. Rio de Janeiro, 2014. Disponível em <http://agenciabrasil.ebc.com.br/economia/noticia/2014-09/> Acessado em 18 Janeiro 2015.

16. INEP. Censo escolar da educação básica 2013.Resumo técnico. Disponível em <http://download.inep.gov.br/educacao_basica/censo_escolar/resumos_tecnicos/resumo_tecnico_censo _educacao_basica_2013.pdf $>$. Acesso em 19 Janeiro de 2015.

17. Unesco. Diretrizes de políticas da UNESCO para a aprendizagem móvel. Acesso em 18 Janeiro de 2015. Disponível em http://unesdoc.unesco.org/images/0022/002277/227770por.pdf

18. Milligan C. Delivering Staff and Professional Development Using Virtual Learning Environments., Heriot-Watt University,. December 1999.

19. Dochev D, Hristov I. Mobile Learning Applications Ubiquitous Characteristics and Technological Solutions. Bulgarian Academy Of Sciences Cybernetics And Information Technologies. vol. 6, no 3, Sofia, 2006

20. Almeida RR, Araújo JR CF. O Uso de Dispositivos Móveis no Contexto Educativo: Análise de Teses e Dissertações Nacionais. Revista tempos e espaços em educação 2013, jun/dez. V. 11, p.25-36,

21. Saldana P. Novo sistema de ensino investe no tablet. Estadão, 2012. Disponível em: http://www.estadao.com.br/noticias/geral,novo-sistema-de-ensino-investe-no-tablet-imp-,974496. Acesso em 20 Janeiro 2015.

22. TUDOCELULAR, Google tem cerca de 1 bilhão de usuários ativos por mês. 2014 Disponível em: $<$ http://www.tudocelular.com/android/noticias/n37543/google-cerca-1b-usuarios-android-ativos-pormes.html> Acesso em 18 Janeiro 2015.

23. Yau, JY-K, Joy MS. A Context-aware Personalized M-learning Application Based on M-learning Preferences. In: 6th IEEE International Conference on Wireless, Mobile, and Ubiquitous Technologies in Education (WMUTE 2010), 2010; 12-16 Apr, Kaohsiung, Taiwan.

24. Clark B. Measuring Performance: the marketing perspective. In: NELLY, A. (org.) Business Performance measurement: Theory and practice. Cambridge University Press: 2002.

25. Souza LFNI. Estratégias de aprendizagem e fatores motivacionais relacionados. Educ. rev. 2010; 36:95107. doi: 10.1590/S0104-40602010000100008.

26. Gugelmin F. Apesar de ter mais downloads, Google Play ainda rende menos que a AppStore. Games dominam as estatísticas. TECMUNDO, 2013. Disponível em: <https://www.tecmundo.com.br/googleplay/46510-apesar-de-ter-mais-downloads-google-play-ainda-rende-menos-que-a-app-store.htm> . Acesso em 20 Janeiro 2015.

27. Silveira SR, BARONE DAC. Jogos Educativos computadorizados utilizando a abordagem de algoritmos genéticos. Universidade Federal do Rio Grande do Sul. Instituto de Informática. Curso de Pós-Graduação em Ciências da Computação; 1998.

28. Zanella AS et al. M-Learning ou Aprendizagem com mobilidade: um estudo exploratório sobre sua utilização no Brasil; 2014.

29. Chabot D. Pedagogia emocional-sentir para aprender. Sá Editora; 2013. 